\title{
ANTARKTISCHE GEBIRGSLANDSCHAFTEN
}

\section{Hans Steinitz}

Ein Blick auf die Karte der Antarktis zeigt, daß an zwei Stellen das Meer in weiten Bögen tief in das Festland hineingreift, die Roß- und die Weddell-See - beide gegen das Land hin durch riesenhafte Eisschelfs, das Roß- und das Filchner-Schelf, blockiert. Nimmt man an, daß diese beiden großen Meerbusen unter der dicken Schicht von $E$ is miteinander verbunden sind, oder daß auch nur eine Tieflandsrinne dort verläuft, das übrige gebirgige und Hochplateau-Land in zwei separate Erdmassen teilend, dann kommt man zu einer natürlichen Zweiteilung der Antarktis; ungefähr zu dem gleichen Ergebnis gelangt man, wenn man den zentralen großen Gebirgszug der Antarktis, den «Antarktischen Horst», als zentrale Scheidelinie ansieht. Das Ergebnis ist in beiden Fällen eine kleinere «Westantarktis», der westlichen Hemisphäre zugehörig und mit der Graham-Halbinsel sich gleichsam an die Südspitze Südamerikas anklammernd, und eine sehr viel größere Landmasse «Ostantarktis», die im großen und ganzen zur östlichen Hemisphäre gehört und aus den riesigen, kompakten und relativ konturlosen Landmassen des afrikanischen und des australischen Quadranten besteht. Diese Einteilung ist recht zweckmäßig, nur sind ihre Namen verwirrend: steht man etwa auf dem Roß-Eisschelf und blickt, wie es sich bei einer geographischen Orientierung gehört, nach Norden, dann hat man die Westantarktis im Osten, und die Ostantarktis im Westen. Ähnliche Konfusionen ergeben sich aus der Lage dieses Erdteils um den Südpunkt der Erdachse herum übrigens immer wieder und in allen Formen, z. B. auch in der Zeitbestimmung. Aber keine dieser Einteilungsformeln hilft uns bei der Kenntnis dieses Kontinents sehr viel weiter. Wie dieser aussieht, ist immer noch sehr wenig bekannt. Die den Kontinent bedeckende dicke Eisschicht, an einzelnen Stellen bis zu dreitausend oder gar viertausend Meter dick, ist für den Eindruck der Konturenlosigkeit verantwortlich, der tür die Antarktis so charakteristisch ist: ein Eisplateau, mehrheitlich flach, stellenweise, namentlich im Zentrum des Kontinents, leicht kuppelartig gewölbt - und gelegentlich von Gebirgszügen oder wenigstens den Gipfeln von Gebirgen, soweit sie über die dicke Eisdecke hinausragen, unterbrochen. Einzelne Berggipfel, die durch die Eisschicht hindurchstoßen, heißen «Nunataks»; sie sind in einzelnen Teilen der antarktischen Landschaft recht häufig und leisten als Erkennungsmarken und Wegweiser und als trigonometrische Punkte wertvolle Dienste. Ohne sie wäre das unendliche gleichmäßige Eisplateau noch sehr viel einförmiger und eintöniger.

Auch die eigentlichen Gebirgszüge ragen häufig nur mit ihren Gipfelspitzen aus dem Eis heraus und verlieren dann den optischen Eindruck alpinen Hochgebirges. Vielfach aber sind sie auch in ihrer ganzen grausamen Pracht sichtbar, nämlich entweder wenn sie sich unmittelbar an den Küsten entlang ziehen, wie vor allem ein großer Teil des Antarktischen Horsts und die Bergketten, die das Rückgrat der Graham-Halbinsel bilden, oder wenn ihre Steiltäler von Gletschern gefüllt werden, die von den Eismengen des Hochplateaus hinunter zur Küste fließen. Die Grundform des Kontinents ist, freilich mit zahllosen Ausnahmen, die eines flachen Tellers mit erhöhtem Rand, wobei allerdings die Tellerfläche erstens, wie gesagt, leicht kuppelförmig gewölbt ist und zweitens dank der dicken Eisschicht ein oftmals $3000 \mathrm{~m}$ hohes Plateau darstellt; der erhöhte Rand besteht aus Hochgebirge, das direkt aus dem Meer aufzusteigen scheint und dessen höchste Gipfel oft genug die 4000-m-Grenze übersteigen. Nach der Innenseite zu senken sich diese Berggipfel und Kämme dann sehr viel weniger, nur bis auf die Plateauhöhe, und wo immer das Plateau über niedrige Bergkämme oder Sattel überquillt, ergießt sich dann ein mächtiger Gletscher zu Tal, d.h.zur Küste, oft genug weit hinaus in das Wasser überhängend.

Diese Erscheinung ist am deutlichsten und eindrucksvollsten ausgeprägt an der Küste des Victoria-Landes, zur Roß-See hinunter, mit den Gebirgszügen der Admirali- 


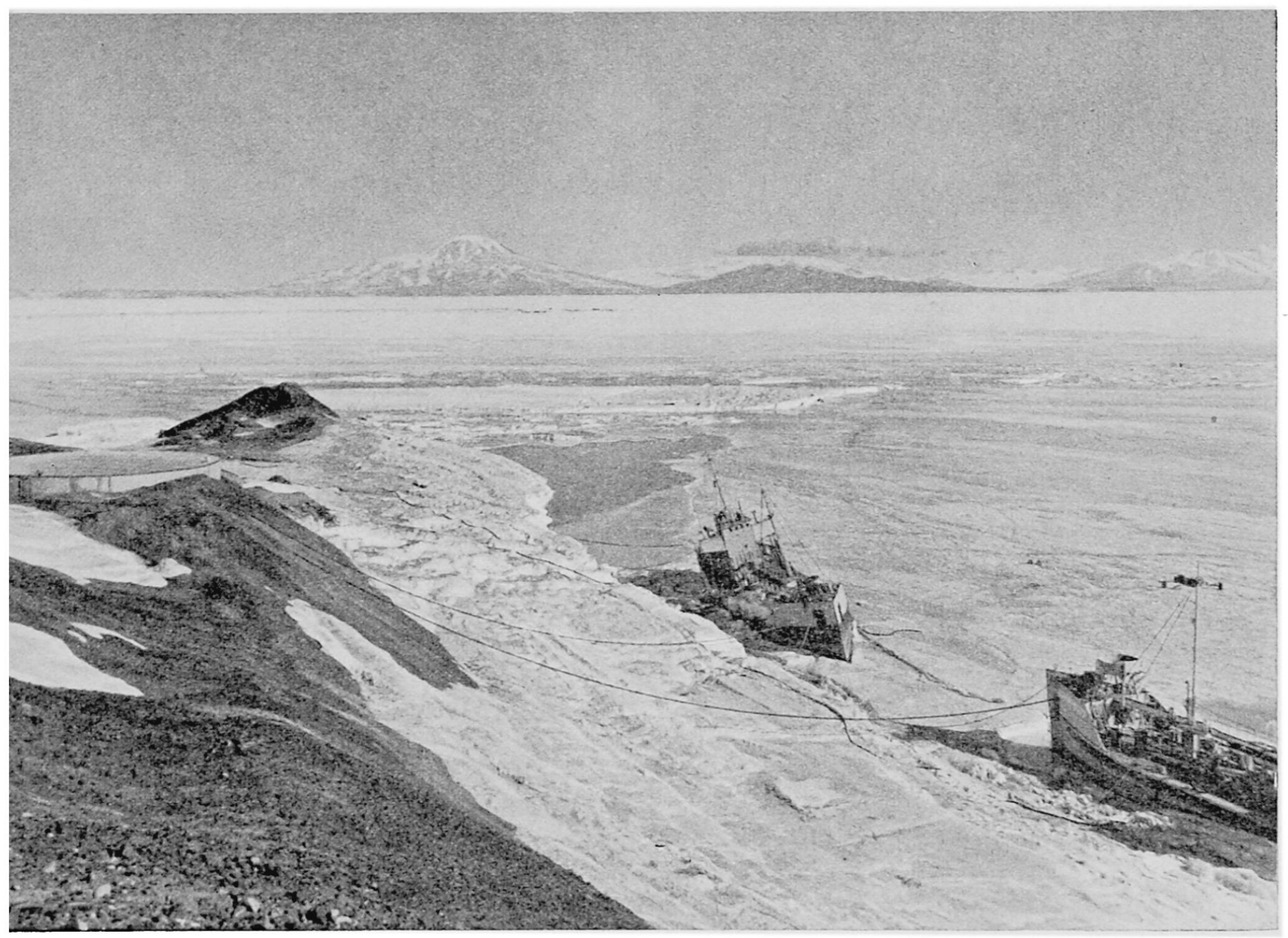

McMurdo Sund. In diesem antarktischen Verkehrsknotenpunkt liegen eingefroren zwei Schiffe. Im Mittelgrund links die dunkle Silhouette des “Observation Hill», im Hintergrund der Kegel des Mount Discovery, eines über $3000 \mathrm{~m}$ hohen erloschenen Vulkans, des letzten Ausläufers des Victoria-Landes.

Photo U. S. NAVY

täts-, Prinz Albert- und Royal Society-Berge, sowie ferner auch an der Westküste des Graham-Landes, bis hinunter zum Filchner-Schelf, und den freilich niedrigeren Gipfeln der Edsel-Ford-Berge an der Sulzberger-Bucht und den Napier-Bergen an der Spitze der Enderby-Halbinsel. Im sogenannten «Neu-Schwabenland»-Gebiet liegt zwischen Küste und Gebirgskamm noch ein schmaler Landstreifen, der aber an dem Bild unseres Tellers mit dem erhöhten Rand im Prinzip nichts ändert.

Der «Große Antarktische Horst», das wichtigste und größte Gebirgsmassiv der Antarktis, gehört mit seinen mehr als $2000 \mathrm{~km}$ Gesamtlänge zu den längsten Gebirgszügen der Erde; vielleicht ist es auch noch viel länger, aber im Innern des MarieByrd-Landes, wo es sich verliert, hat man es noch nicht erforschen können und kennt daher noch nicht seine gesamten Dimensionen. Ob die kürzlich entdeckten Horlickund Sentinel-Berge oder auch die mächtige Forrestal-Kette im Edith-Ronne-Land zu dem Horst gehören, ist zweifelhaft, sollte es aber der Fall sein, dann hätten wir es hier mit einer unerhört wilden und gewaltigen Gebirgskette zu tun, die als Rückgrat des Kontinents diesen von einer Küste zur anderen durchzieht. Der «Horst» hat im Grunde keinen rechten Namen, die Bezeichnung «antarktischer Horst» ist lediglich eine Erklärung seines wahrscheinlichen geologischen Ursprungs. Der «Horst» ist wenigstens in seinem zentralen Teil, vom Kap Adare an der Spitze des Victorialandes 
bis zu dem von Amundsen für seine Polarbezwingung durchquerten Axel-HeibergGletscher, relativ gut erforscht, und die meisten hohen Gipfel haben nicht nur Namen, sondern sind auch ihrer wenigstens ungefähren Höhe nach bekannt. Der Mount Markham mit seinem charakteristischen Doppelgipfel ist von den Vorstößen von ScotT und Shakelton über das Roß-Schelf zum Südpolplateau her ein alter Bekannter der Antarktisforschung: mit seinen 4600 Metern galt er bis vor kurzem als höchster Gipfel des ganzen Kontinents, ist aber durch die Entdeckung von mehreren Gipfeln über $5000 \mathrm{~m}$ Höhe in der Westantarktis, deren Messung freilich noch nicht bestätigt ist, entthront worden. Andere höchste Gipfel der verschiedenen Ketten des «Horsts» sind u. a. Mount Wade (4570 m), Mount Kirkpatrick (4250 m), Mount Lister (3900 m) und Mount Sabine $(3650 \mathrm{~m})$.

Zwischen diesen Gipfeln und den an sie grenzenden niedrigeren Spitzen und Kämmen ergießen sich in fast regelmäßigen Abständen Gletscher talwärts, darunter die bekanntesten Riesengletscher der Antarktis wie der Beardmore, der Scott- und der Amundsen-Gletscher, der Nimrod-, Axel-Heiberg-, Koettritz- und Ferrargletscher: zumeist Eisströme von unvorstellbaren Dimensionen, mit den uns geläufigen Gletschern nach Länge, Breite und beförderten Eismassen in keiner Weise vergleichbar. Einige sind über $200 \mathrm{~km}$ lang, einige mehr als $60 \mathrm{~km}$ breit, einige bringen ihr Eis mit einer Tagesgeschwindigkeit von fünf Metern zutal. Über die Eismenge eines solchen Gletschers gibt es nur rohe Schätzungen: Ziffern von hundert Milliarden Kubikmetern klingen durchaus glaubwürdig, übersteigen aber das menschliche Vorstellungsvermögen.

Der zweite wichtige Gebirgszug der Antarktis ist ein Faltengebirge, das durch das Grahamland läuft und am Filchner-Schelf endet. Es ist vermutlich erdgeschichtlich eine direkte Fortsetzung der Anden, die ja durch ganz Südamerika gehen und erst am Kap Horn abbrechen: jenseits der Drakestraße wieder auftauchend, setzt es sich entlang der ganzen Graham-Halbinsel bis zum Elsworth-Hochplateau fort und zeigt eine geologische Verwandtschaft mit den Anden, die zu dem Schluß führt, daß die Drakestraße nur ein relativ später Erdeinbruch ist, die beiden Kontinente also ursprünglich zusammengehangen haben. Andere Gebirgszüge von Bedeutung sind die Gebirgsgruppen im Neu-Schwabenland, vor allem die Mühlig-Hoffmann-Kette, und die verschiedenen Gebirgszüge im Marie-Byrd-Land wie die Edsel-Ford-, Rockefeller- und «Executive Committee»-Massive. Auch diese Gebirge haben alle ihre Gipfel an oder über der 4000-m-Grenze: zwei isolierte Spitzen nordwestlich der Executive CommitteeKette, Mount Vinson und Mount Nimitz, sind sogar provisorisch und noch unbestätigt auf 6000 und $4800 \mathrm{~m}$ Höhe geschätzt worden.

Der Felsboden, soweit man ihn aus Gebirgen, Nunataks und den wenigen eisfreien Bodenproben kennt, besteht vorwiegend aus Granit, Gneis, Beacon-Sandstein und gelegentlich auch Basalt. Geologen glauben, daß der ganze Kontinent erdgeschichtlich sehr alt ist, also bereits in einer früheren Erdperiode geformt wurde. Höchst bemerkenswert sind verschiedene Funde fossilierter Tier- und Pflanzenreste, darunter sogar tropischer Farnkräuter: unwiderleglich geht daraus hervor, daß zu irgend einem Zeitpunkt das Klima der Antarktis gänzlich anders gewesen sein muß.

Überaus seltsam ist der vulkanische Charakter eines Teiles des antarktischen Felsenbettes, eines der vielen Naturwunder der antarktischen Welt. Vielfach, auf den Inseln vor dem Grahamland wie auch auf dem eigentlichen Festland, z. B. am McMurdo-Sund, besteht der Boden aus erstarrtem porösen Lava. Die Krater erloschener Vulkane sind in der Antarktis sogar durchaus nicht sehr selten, aber was den Laien besonders erstaunt, sind die noch lebenden Vulkane, die man an einigen Stellen der Antarktis gefunden hat. Der bekannteste von ihnen ist der schon vor 120 Jahren entdeckte Mount Erebus auf der Roßinsel, der den Eingang zum McMurdo-Sund beherrscht, ein $4200 \mathrm{~m}$ hoher Berg von klassisch-regelmäßiger Kegelform, vollkommen 


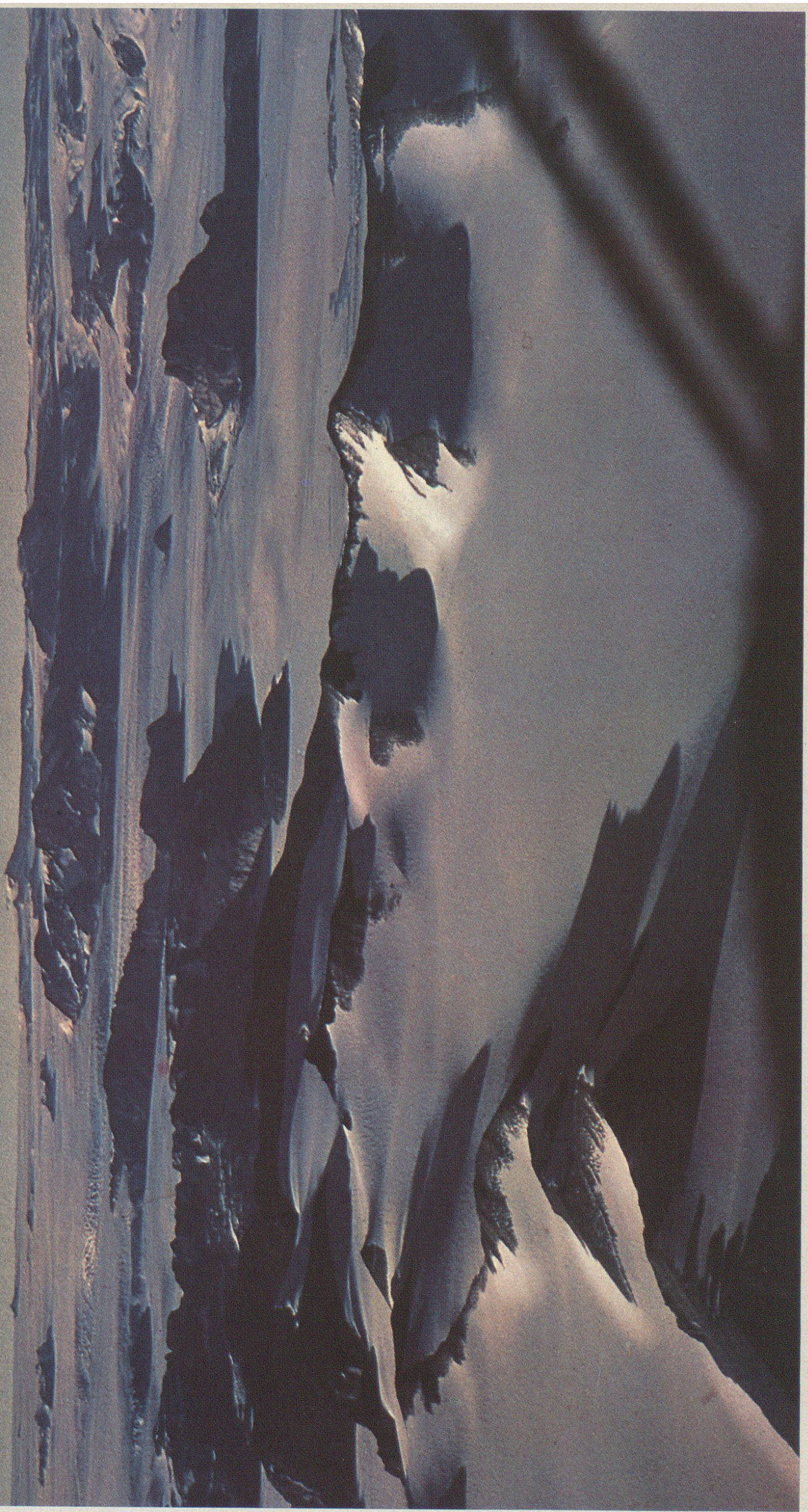

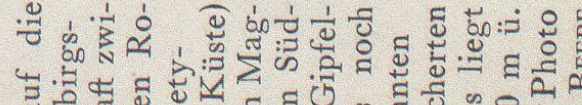
㟧击

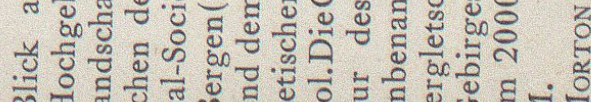


eis- und schneebedeckt, der sich zu seiner stattlichen Höhe direkt vom Meeresspiegel aus erhebt und aus dessen Kraterspitze ständig eine Rauchfahne aufsteigt: sicherlich eines der ergreifendsten und erhabensten Naturschauspiele, die sich auf Erden finden lassen.

$\mathrm{Ob}$ es noch andere aktive Vulkane in der Antarktis gibt, ist nicht ganz sicher; dagegen gibt es zum mindesten drei Inseln direkt vor den Küsten des Festlandes, deren vulkanischer Charakter sich in großer Bodenwärme (daher eisfreier Boden und eisfreie Hafenbucht) und in Schwefeldämpfen, die aus Erdspalten aufsteigen, manifestiert, so Deception in den Süd-Shetlands, deren Hafenbucht in der Periode der frühen Antarktiserschließung eine wichtige Rolle spielte und die kleine Insel Zavodowski, die nördlichste Insel der Süd-Sandwich-Gruppe; auch die Lichtmeß-Insel, ebenfalls zur SüdSandwich-Gruppe gehörig, sendet Schwefeldämpfe aus dem Erdboden über das Meer.

Außer den wenigen Stellen, in denen aktive Vulkankräfte für eine natürliche Bodenheizung sorgen, gibt es noch einige andere Stellen in der Antarktis, wo - abgesehen von den Steilhängen des Hochgebirges - kein Eis vorhanden ist und der natürliche Boden frei liegt. Sinngemäß nennt man diese Stellen, obwohl sie kahle Felsen darbieten mit nur minimalen Spuren von Vegetation, «Oasen», weil sie zur umgebenden Eiswelt einen ebenso dramatischen Kontrast bilden wie die grüne, echte «Oasis» zu den Sandwüsten anderer Erdstriche. Schon Scotr entdeckte 1903 jenseits der «Horst»-Berge, in der Luftlinie nicht weit vom McMurdo-Sund, eine solche Oase, die er schlicht «Dry Valley», trockenes Tal, taufte; spätere Expeditionen entdeckten noch die eisfreien «Vestford Hills», die «Schirmacher Oasis» in der Mühlig-HoffmannBergkette, und vor allem die «Bunger Oasis», vermutlich die größte von allen, dicht hinter der Knox-Küste. Alle diese Oasen befinden sich nahe den Küsten in der Ostantarktis; sie enthalten Binnenseen, die wenigstens im antarktischen Sommer eisfrei sind, freundliche kleine Bäche, die von den umliegęnden Gletschern oder Firnen herabrieseln, und trockene Moränen, die erkennen lassen, daß an diesen Stellen die antarktische Eisdecke zurückgewichen ist. $\mathrm{Ob}$ die Seen aus geschmolzenem Schnee und Eis gespeist werden oder von Meerwasser, das der Wind landeinwärts geweht hat, ist nicht ganz klar; dagegen besteht Grund zu der Annahme, daß diese Oasen nicht oder nicht nennenswert wachsen, also von einem allgemeinen kontinuierlichen Gletscher- und Eisschwund kaum die Rede sein kann. Die eisfreien Oasen scheinen jedenfalls in den letzten Jahrzehnten Form und Umfang nicht geändert zu haben.

Aus: Der 7. Kontinent. Das Ringen um die antarktische Eiswelt. Geogr. Verlag Kümmerly und Frey, Bern.

\title{
F R I BOURG
}

\section{ET LE CONGRÈS DE L'ASSOCIATION SUISSE DES PROFESSEURS DE GÉGRAPHIE}

\author{
Jean-Paul Moreau
}

\begin{abstract}
Tenant cette année à Fribourg son congrès annuel, l'Association Suisse des Professeurs de Géographie avait prévu une visite de la ville, organisée par le Directeur du Musée d'Histoire Naturelle, Dr. O. Büchi, et le Directeur de l'Institut de Géographie, Prof. Dr. J.-P. Moreau.

Les visiteurs firent d'abord halte au Pont de Pérolles. De ce belvédère au-dessus du célèbre canyon de la Sarine, M. Büchi montra le soubassement molassique constituant l'essentiel des parois verticales du canyon, mais aussi les cailloutis glaciaires qui couvrent par endroits la molasse révélant l'existence, lors des diverses glaciations, de lits successifs de la Sarine, différents de l'actuel tracé.

Un peu avant Marly-le-Grand, M. Büchi exposa en quelques mots la topographie de cette cuvette à fond plat, isolée par des gorges à l'amont comme à l'aval: là aussi, les épisodes gla-
\end{abstract}

